\title{
Für sieben KVen könnte es eine kräftige Honorarspritze geben
}

— Der im Juli 2015 eingeführte $\$ 87 \mathrm{a}$ Abs. 4a SGB V sieht vor, dass in manchen $K V e n$ noch in diesem Jahr über eine einmalige basiswirksame Honorarerhöhung verhandelt werden soll, die dann 2017 in Kraft tritt. Dafür muss in der betroffenen $\mathrm{KV}$ die morbiditätsbedingte Gesamtvergütung (MGV) je Versicherten für das Jahr 2014 unter dem Bundesdurchschnitt gelegen haben. Das Institut des Bewertungsausschusses (InBa) hat nun sieben KVen identifiziert, die deshalb 2017 einen deutlichen Zuschlag zu den normalen Anhebungen der MGV bekommen müssten.

\section{MMW-KOMMENTAR}

Das Gesetz sieht zwar nur eine einmalige Erhöhung des Aufsatzwerts vor, von dem aus die Honorarverhandlungen starten - die bisher zu kurz gekommenen KVen dürften sich trotzdem freuen. Interessant wird dabei eine
Tab. 1 Potenzielle Anhebung der morbiditätsbedingten Gesamtvergütung (MGV) für 2017 (in Millionen Euro)

\begin{tabular}{|c|c|c|}
\hline KV & MGV & Zuwachs \\
\hline Hamburg & 492,61 & 39,33 \\
\hline Westfalen-Lippe & $2.336,88$ & 41,54 \\
\hline Hessen & $1.700,15$ & 75,49 \\
\hline Nordrhein & $2.643,76$ & 10,79 \\
\hline Baden-Württemberg & $2.671,68$ & 39,43 \\
\hline Schleswig-Holstein & 814,77 & 16,45 \\
\hline Sachsen-Anhalt & 707,44 & 9,55 \\
\hline
\end{tabular}

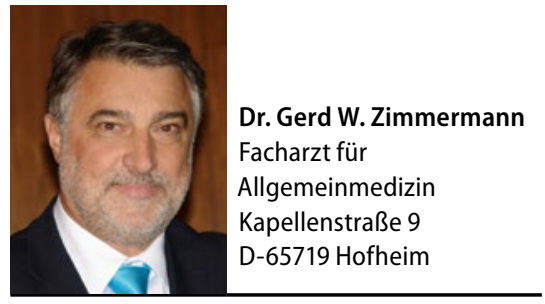

weitere Auflage des Gesetzgebers: Eine Anhebung ist nur dann angesagt, wenn in den Verhandlungen festgestellt wird, dass der Aufsatzwert im Jahr 2014 unbegründet zu niedrig war. Schafft es die KV, dies nachzuweisen, muss der Aufsatzwert für 2017 im gleichen Umfang erhöht werden. Dabei darf durch die Erhöhung nicht der Bundesdurchschnitt des Jahres 2014 überschritten werden. Die Erhöhung kann auch schrittweise über mehrere Jahre verteilt werden. Die zusätzlichen Mittel müssen zur Verbesserung der Versorgungsstruktur eingesetzt werden. Umverteilungen zulasten anderer KVen müssen ausgeschlossen werden.

Die neu gewählten KV-Vorstände stehen damit gleich unter erheblichem Zugzwang. In Hessen z. B. würde eine vollständige Realisierung der gesetzlichen Vorgabe die MGV um 75,49 Millionen Euro anheben, in Hamburg um 39,33 Millionen Euro (Tab. 1). Nur wenn dieser einmalige Aufschlag gelingt, sind dort auch in den Folgejahren zumindest bundesdurchschnittliche Honorare zu erwarten. Die bisher besser gestellten KVen können sich dabei beruhigt zurücklehnen, denn wegnehmen darf man ihnen nichts.

\section{Ausnahmen von der Plausi-Prüfung nach Zeit}

\author{
— 106a SGB V verpflichtet Kranken- \\ kassen und KVen, Rechtmäßigkeit und \\ Plausibilität der vertragsärztlichen Ab- \\ rechnungen zu prüfen. Das konkrete \\ Prüfungsverfahren haben die Partner \\ auf Bundesebene in Richtlinien festge- \\ legt. Danach werden Tages- und Quar- \\ talsprofile auf der Basis der abgerechne- \\ ten Nrn. in Verbindung mit den zugehö- \\ rigen Prüfzeiten gemäß Anhang 3 des \\ EBM erstellt.
}

\section{MMW-KOMMENTAR}

Bei der Erstellung der Zeitprofile werden Leistungen nicht berücksichtigt, wenn sie im organisierten Notfalldienst erbracht und auf dem Vordruck-Muster 19 abgerechnet wurden, wenn sie im Zusammenhang mit einer unvorhergesehenen Inanspruchnahme des Vertragsarztes außerhalb der Sprechstundenzeiten erforderlich werden oder wenn sie eine Unterbrechung der Sprechstunde und Verlassen der Praxis erforderlich machen.
Auch unverzüglich nach Bestellung durchgeführte, dringende Besuche gehen nicht ins Zeitprofil ein.

Die Auffälligkeitsprüfung ist dann einfach. Summieren sich die Prüfzeiten an mindestens drei Tagen des Quartals auf mehr als 12 Stunden oder im Quartalsprofil auf mehr als 780 Stunden, werden ergänzende Abrechnungsprüfungen eingeleitet. Von deren Ergebnis ist es abhängig, ob ein Prüfverfahren eingeleitet wird oder nicht. 